\title{
Expanding Access To Rural Healthcare Services And Benefits Through Secure, Interactive Video Links: A Case Study Of Video Claims Taking Implementation In The Great Plains
}

Dana Michael Harsell, University of North Dakota, USA Christine Carlascio Harsell, University of North Dakota, USA

Robert S. Wood, University of North Dakota, USA

\begin{abstract}
Context: Between 2003 and 2008, the University of North Dakota Center for Rural Service Delivery (RSD) increased access to a number of vital government services for citizens in rural communities and Native American reservations through the Video Claims Taking (VCT) program, which allowed claimants in rural healthcare settings to apply for benefits over secure interactive video networks to distant government offices. VCT helped increase healthcare reimbursement, social and related public service benefits to 31 underserved communities in six states and leveraged a significant increase in applications for government services and monetary benefits to these communities. Methods: A case-study examines the implementation, use and impact of the VCT technology over the RSD's five year operation. Findings: The authors offer five principal findings which center on the relationships between government agencies and rural claimants, some cultural considerations posed by this electronic form of service delivery, the program's potential to realize certain efficiencies, the technical challenges faced during implementation and overcoming a number of organizational and communications barriers. Conclusions: The foregoing analysis of the RSDs initiative and its VCT program contributes to a better understanding of how to leverage an interactive video platform to provide government healthcare reimbursement services to underrepresented groups in rural contexts for public agencies and private healthcare providers that are interested in adopting a similar model of service delivery to their stakeholders
\end{abstract}

Keywords: Rural Healthcare; Telemedicine; Implementing Telemedicine; Video Claims Taking; Medicare; Medicaid; Indian Health Service; Social Security; Veterans Benefits

\section{INTRODUCTION}

$\mathrm{n}$ health care policy over the last decade, e-government initiatives have encompassed efforts to expand access to reimbursed services by developing websites where stakeholders can gather healthcare information and electronically access requisite forms and applications for healthcare services and reimbursement. However, the assumption that e-government initiatives can provide universal citizen access to health care reimbursement benefits is challenged by the aptly termed "digital divide." Put simply, large segments of the population still have little or no access to the internet and may lack the requisite education or computing skills required to participate as a stakeholder in e-government. Indeed, certain aspects of these e-government initiatives have the potential to further disadvantage those groups that are already marginalized by society (Silcock, 2001). In this context, the digital divide serves as a stark reminder of those who are able to leverage benefits through 
technological means and those who can or will not. Ostensibly, this divide impacts the poor, the undereducated and those living in rural communities (US GAO, 2006). Nowhere is this more evident than in the administration of rural health benefits.

It is also widely acknowledged that residents of rural communities face a number of barriers to health care services, more generally. People living in rural settings tend to be older and poorer than their urban counterparts and statistically, those living in rural households experience higher mortality, disability and chronic disease rates. Although rural areas account for nearly 25 percent of the US population, fewer than 9 percent of all active physicians practice in these settings (Gamm, et. al., 2003). US Census Bureau data also suggest a pattern of broader depopulation trends among rural areas; Great Plains states are especially affected by rural flight (Carr \& Kefalas, 2009). So often these patterns of out-migration create situations where the older and less educated remain.

Expanding enrollment and adequate access to Medicare, Medicaid, Veteran, and IHS reimbursed healthcare services (through traditional or electronic means) to residents of underserved rural areas remain a perennial challenge for government entities and health care providers alike. Socioeconomic hardships experienced by rural residents pose one such barrier (Felix \& Stewart, 2003). Low income and minority populations often face additional burdens when trying to coordinate these services, not the least of which is complying with the paperwork that is associated with the process (Stevens, Seid \& Halfon, 2003). Persons with reported health problems, certain minorities and those with less education were more likely to perceive obstacles in enrolling in Medicaid (Stuber \& Bradley, 2005). Moreover, "complex eligibility criteria and onerous application forms and processes" may inhibit families from enrolling in public programs (Stevens, Seid \& Halfon, 2003). Eligible Native American populations experience similar barriers when enrolling for Medicare and Medicaid (US GAO, 2008).

This study examines the Rural Service Delivery (RSD) initiative and its efforts to plan, implement, and maintain the Video Claims Taking program (VCT); this technology allows claimants in underserved rural areas to apply for public health and social services benefits over a secure video conferencing network when visiting a local hospital or a designated terminal station. More specifically, the case-study considers the provision of Social Security Administration (SSA), Indian Health Services (IHS), Veterans Affairs (VA) and related benefits over these secure video networks to claimants in locations where the presence of full-time human service providers and case coordinators cannot be justified from an economic standpoint. From this case-study, the authors are able to explore a number of findings centering on RSD's role in the implementation and administration of the VCT program and to illuminate successes and challenges faced over its five year run.

\section{HISTORY OF THE RURAL SERVICE DELIVERY PROJECT}

In 2003, Congress earmarked funds to be spent by the Government Rural Outreach Initiative in partnership with the University of North Dakota Center for Rural Service Delivery (RSD) to develop a program to increase access to services for citizens in underserved rural communities and Native American reservations. The RSD was charged with implementing, troubleshooting and managing an infrastructure needed for the beneficiaries of health and certain health-related programs to work directly with caseworkers over secure video conferencing connections among states in the Great Plains region, including: North Dakota, South Dakota, Montana, Minnesota, Wyoming and Utah. Additional funding for the Initiative came from the SSA with an interagency agreement from the General Services Administration (GSA). Federal funding expired in 2008.

The RSD brings people in rural areas and in Native American reservations closer to government services via secure, dedicated interactive video links. Put simply, Video Claims Taking bridges the distance by placing citizens in direct contact with distant government service providers from locations within their own community. In its five years, 31 Rural Service Delivery locations were established in North Dakota, Minnesota, Montana, South Dakota, Utah and Wyoming (see Table 1). As an organization, the mission of the North Dakota Center for Rural Service Delivery included:

project management, institutional development and training, organizational communication, telecommunications connectivity and support, equipment installation and operation, network and service management, and evaluation 
and impact assessments. The RSD staff [also] serves as a necessary supplement to the otherwise overcommitted SSA and IHS staffers for the installation, operation, troubleshooting, and evaluation of VSD sites (RSD, 2007, p. 8-9).

Levels and types of services established through the RSD initiative vary among communities; the VCT links have been used to help citizens apply for health care benefits, income supplementation, veteran's benefits and prescription assistance. The RSD worked directly with the Social Security Administration to link 18 communities to 11 regional Social Security offices in six states. These VCT locations allow those in need to more easily apply for disability, supplemental income and elder citizen benefits. For instance, Pine Ridge, SD residents can access services either by appointment or "on demand" via a "virtual window" from SSA caseworkers located 110 miles away in Rapid City, SD.

The flexible nature of VCT service delivery also allows for better coordination of services provided by crosscutting programs. In North Dakota, dedicated video links connect a dental care provider, an IHS hospital and a distant Social Security office, which greatly streamlines the time required to file and process claims. To the West, VCT terminals interlink Montana Job Services, the Human Development Service Center and the Public Health Services Hospital together in order to provide eligible rural and reservation citizens with services from all three locations.

Table 1: RSD Video Claims Taking Locations ${ }^{\mathrm{a}}$

\begin{tabular}{|l|l|}
\hline North Dakota & Minnesota \\
\hline SSA Office & SSA Office \\
\hline SSA Office Minot, ND & SSA Office (2 multi-state points of service) Fergus Falls, MN \\
\hline SSA Office Bismarck, ND & Montana \\
\hline DDS Office Bismarck, ND & SSA Office \\
\hline Community Site & SSA Office Great Falls, MT \\
\hline Belcourt PHS Indian Hospital Belcourt, ND & SSA Office Glasgow, MT \\
\hline Dickinson Public Library Dickinson, ND & Community Site \\
\hline St Joseph's Hospital Dickinson, ND & NE Montana Job Service Center Glasgow, MT \\
\hline Mercy Hospital Devils Lake, ND & Human Resources Resource Council Havre, MT \\
\hline South Dakota & Fort Belknap PHS Indian Hospital Harlem, MT \\
\hline SSA Office & Browning PHS Indian Hospital Browning, MT \\
\hline SSA Office Rapid City, SD & Wyoming \\
\hline SSA Office (2 points of service) Huron, SD & SSA Office \\
\hline SSA Office Aberdeen, SD & SSA Office Casper, WY \\
\hline Community Site & Community Site \\
\hline Rosebud PHS Indian Hospital Rosebud, SD & Fort Washakie PHS Indian Health Center Fort Washakie, WY \\
\hline Pine Ridge PHS Indian Hospital Pine Ridge, SD & Utah \\
\hline Kyle PHS Health Center Kyle, SD & SSA Office \\
\hline Wanblee PHS Health Center Wanblee, SD & SSA Office Ogden, UT \\
\hline Eagle Butte PHS Indian Hospital Eagle Butte, SD & Community Site \\
\hline Todd County Building Mission, SD & Division of Services for People with Disabilities Logan, UT \\
\hline Capital University Center Pierre, SD & \\
\hline St Mary's Hospital Pierre, SD & \\
\hline South Dakota Career Center, Mobridge, SD & \\
\hline a Aapt From: Univers of Norh Dakt Rut Service Delivery, 2007
\end{tabular}

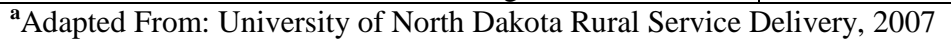

\section{METHODS AND DATA}

Case studies are well suited for the observation of contemporary empirical phenomena (Yin 2003a), understanding "bounded systems" or for when the lessons are not always apparent to an outside observer (Stake, 1978). This inquiry utilized a descriptive case study method and the unit of analysis consisted of the RSD implementation process (see Yin, 2003a) within a larger network of rural healthcare clinics and government agencies. When a case analysis is grounded in a broader research literature, its lessons can "advance knowledge and understanding of a given topic" (Yin, 2003b, p. 3). 
The analysis of the RSD implementation process is important because the lessons learned stand to offer valuable insights into the opportunities, benefits and challenges that await any government agency, healthcare provider or third party who are interested in providing similar models of service delivery to their stakeholders. Moreover, Daryl West offers a sensible justification for this type of case study:

Because it is impossible to know whether a particular technological innovation will produce large-scale or smallscale change until years have passed, it makes sense for researchers to focus on the nature and direction of new practices in the short run. The virtues of studying short term changes is that it provides insights about longer-term shifts and gives policy makers benchmarks for evaluating how close they are to achieving particular goals and outcomes $(2004,16)$.

In the case study approach, data are often collected from greater than one source or perspective (Yin, 2003a). Data for this case were obtained through a mixture of elite interviews and from RSD reporting documents produced for both public and in-house consumption. Elite interviews were conducted with 5 key people involved with the project; they were selected for their extensive knowledge of RSD implementation from the perspectives of the Social Security Administration and Indian Health Service field offices, rural healthcare clinics and RSD project management. These in-depth interviews were guided by a set of open-ended protocols, but remained semistructured to maximize the flow of information between the participant and the interviewers (see Aberbach and Rockman, 1989). The nature of the video service delivery network also allowed the authors to conduct comprehensive "face-to-face" interviews over the VSD network. Analyses of documents and interview data were performed to construct an analytic framework or "case description" in order to better explain aspects of the implementation process (see Yin, 2009).

\section{FINDINGS AND DISCUSSION}

The case description reveals five principal findings from the RSD's implementation of Video Claims Taking and its five year effort to improve the coordination of healthcare and social services reimbursement to stakeholders in rural areas. First, the transactional nature of video service delivery has a number of demonstrable advantages over other forms of electronic (even telephone based) delivery methods, not the least of which is breaking down adversarial relationships between caseworker and claimants and fostering advocacy. Second, agency and healthcare providers in this study have identified and adjusted for a number of cultural considerations related to Video Claims Taking. Third, the VCT model helps to streamline rural applications, increase the quality of initial applications, and reduce the incidence of costly appeals. Fourth, RSD deployment faced a number of technical, implementation and deployment challenges, especially in more remote locations. Finally, during the planning and implementation stages, the RSD had to negotiate a number of organizational and communication barriers, including discussions among agencies regarding resources allocation. The following sections discuss these findings in more detail.

\section{Finding One: Improved Relationships, Advocacy And Community Outreach}

The VCT model can help break down adversarial relationships between government and claimants, fosters a sense of advocacy by the claimant and promotes community outreach. Much of the growth of e-government has led to an increase in the range and types of online transactions that can be conducted over the Internet. Egovernment has opened government to many people, however certain cultural, economic and generational barriers can frustrate efforts to provide services to those in need; these barriers may be more pronounced in poor, rural areas.

The face to face transactions over the VCT service delivery model has also demonstrated a number of advantages over traditional service delivery methods (such as postal or telephone based) and other technology or web-based service delivery models. Many eligible residents in underserved communities are unable or unwilling to seek services or submit claims over a web interface. Residents may find residential internet access limited or cost prohibitive and the process of initiating telephone contact with government service providers may be daunting. Moreover, existing web-based service provision models lack the same levels of advocacy and support that a human caseworker can provide for their clients. A North Dakota Center for Rural Service Delivery publication notes that one Native American population in particular: 
has experienced barriers associated with each of the conventional avenues of government service. Few have all of the skills necessary and the personal inertia to see a paper application through to completion. Most members do not have easy access to a telephone. Many tend to distrust communication that is not face to face, particularly communication involving non-Indians [and] the nearest [SSA] office is 121 miles from the reservation (RSD, 2007, p. 3).

Moreover, Silcock rightly argues that the e-government "relationship is no longer just a one-way 'us versus them' proposition; rather, it is about building a partnership between governments and citizens" (2001, p. 88). One SSA employee felt that too often, stakeholders initially assume that they will be entering into an adversarial relationship with his or her caseworker (personal interview). To this end, he reported that the VCT model "tears down" the perception of an adversarial relationship and helps to build relationships of trust much faster than when similar transactions are conducted over the telephone or via other web-based formats.

In terms of outreach, the RSD model has successfully established its presence in some of the poorest rural areas and generated benefits for deserving people. In about 5 years, the number of persons receiving benefits in Jackson County, South Dakota increased from 25 to over 125. Centered in the Badlands, Jackson County is larger than the state of Rhode Island. Before the VCT program, clients in Kyle, SD would have to drive 45 miles to Pine Ridge, SD or 90 miles to Rapid City, SD to meet with a case worker. In many cases, telephones or computers were not a viable option; many households did not have telephone or internet service and cell phone coverage was described as sporadic at best. The efficacy of VCT as an alternative mode of service provision has demonstrated great success in terms of benefits disbursed and in increased numbers of enrollments. For instance, the three VCT sites in the Pine Ridge Indian reservation facilitated over 900 completed applications, which helped to leverage approximately $\$ 8.7$ million dollars in new benefits over this 5 year period.

Agency personnel and healthcare providers also identified ways for the VCT technology to reach out to additional populations. For instance, the technology was used to supplement the Telephone Typewriter (TTY) device that is often used by the hearing impaired. One field office conducted three way video conferences for hearing impaired clients that included a distant American Sign Language interpreter. Future applications of this technology could be adapted to provide services for Community Violence Intervention Center (CVIC) clients and increased access to mental health care that would otherwise be inaccessible in rural areas.

Health care provision can become especially complex among Native American populations who receive benefits through the IHS, which has become a vital physical and mental health care provider for rural Native American populations:

Approximately 55\% of American Indians and Alaska Natives living in the United States rely on the Indian Health Service to provide access to health care services in 49 Hospitals and nearly 600 Other Facilities operated by the IHS, Tribes, and Alaska Native corporations...(US IHS, 2006).

Indian Health Services relies on a patchwork of federal and state funding sources and grants to operate and many of its medical services are funded through by the SSA in the form of Medicare reimbursements, disability or other distributions. To this end, the VCT model has established an important measure of community outreach in these populations and a significant tool to coordinate and leverage available benefits through the IHS.

\section{Finding Two: Cultural Considerations}

Agency personnel and healthcare administrators had to account for a number of cultural considerations related to video service delivery and incorporated these considerations into their VCT strategies. One of the challenges faced by the RSD was designing and implementing a user-friendly service delivery model that can reach those in poor, rural areas. Designing a model that would be used in rural settings was important because although "the internet accounts for much of the ' $\mathrm{e}$ ' in e-government, governments should not make the mistake of assuming that most citizens want and, more importantly, will use government services online" (Silcock, 2001, p. 93). Caseworkers found that a small percent of claimants will not use the VCT terminals. One caseworker from the SSA reported that she travels to staff a contact station to answer questions about once per month and observed that in 
some cases, clients might wait 3-4 hours to speak with her when they could have probably accomplished the same business more quickly over the VSD terminal at the same contact station. To help promote VCT use, this caseworker actively encourages clients to utilize VCT for IHS service reimbursement, but always offers them the choice of traveling to her office, conducting business over the telephone or over the VCT network. After explaining the benefits of the VSD network, many of her clients opt to schedule a VCT appointment.

Technological "solutions need to be sensitive to the social contexts of service use and delivery if they are to be introduced successfully" (Silcock, 2001, p. 93). Indeed, one caseworker discussed how mindful the SSA and IHS field offices have been of their clientele when establishing VSD services, as well as the often delicate contexts they must negotiate to serve their clients. For instance, they were reluctant to place video terminals for general SSA or IHS services in welfare offices because they have learned that many of their clients are sensitive to being seen entering or leaving a welfare office.

\section{Finding Three: Efficiencies}

Application assistance over the Video Service Delivery network streamlines the application process, increases the quality of claims and decreases the incidence of costly appeals. The VCT program has provided claimants with the resources to submit more accurate and more complete claims. A case manager for a rural health clinic in North Dakota (personal interview) echoed this finding; she reported that "forms are such a barrier" for many claimants attempting to get connected with available resources and that required information is more likely to be submitted correctly when forms are filled out while an "advocate" is present. Claimants who fill out paperwork in the virtual presence of a caseworker are able to address specific questions more easily than over the telephone or over a website; claimants can even solicit help by placing the form in front of the camera and pointing to specific sections where they are having difficulties. Frequently, claims were completed during a video conference between Social Security and IHS while the claimant was sitting with an IHS caseworker, resulting in more complete applications and reducing the number of times a Social Security caseworker had to follow-up for additional information, such as additional medical records. Reducing the number of follow-ups required to generate a complete and quality application can also significantly shorten the application process from beginning to end and decrease the lag time claimants must wait to receive benefits or that healthcare providers must wait for reimbursement.

High quality applications can also help reduce the number of applications that are denied (as a result of incomplete information or documentation) and the need for time consuming and costly reconsiderations and appeals. One participant (personal interview) estimated that the costs of the reconsideration process alone totals about seven hundred dollars and the costs of a full review can approach three to four thousand dollars. This technology has the potential to break a cycle where claimants file claims and fail to go through the reconsideration or appeals process when it is denied (opting instead to re-file the claim after a specified waiting period); it is not uncommon for a claimant to re-file the same claim five or six times without ever opting to appeal the decision.

The VCT model provides claimants in remote, rural locations a level of service that is comparable to that of claimants who visit field offices or other physical locations. For example, in the SSA, almost everything that caseworkers can do in person can also be completed over the VSD network. One exception includes applications for new or replacement Social Security cards since the law requires special techniques to verify the authenticity of supporting government forms of identification. For most other types of transactions, it is sufficient for caseworkers to verify a claimant's identity by visually examining government issued identification over the video network. Another SSA caseworker (personal interview) noted that federal attestation laws were modified so claimants can attest to the accuracy of their claim over the VCT network rather than sending a paper form to her office.

\section{Finding Four: Technical And Implementation Challenges}

Video Claims Taking deployment posed a number of implementation and technical challenges that ranged from problems with third party internet service providers to challenges with consistent and quality technical support to maintain the video service links. From a technical standpoint, the increase in front end services (electronic links to the public) had to accompany an increase in back end capacity (personnel and technical support) in order to offer consistent levels of service over the video network. Early planning discussions centered on whether broadband 
connections should utilize cable, DSL or a dedicated T1 line, as the remoteness of the service areas limited the number of viable choices. The rural character of the service areas also posed a challenge in finding and contracting reliable technology support. Government field offices generally have their own tech-support personnel but they were sometimes reluctant to service the VCT equipment and technical support for remote locations was typically contracted out.

Securing transactions and protecting the privacy of the client is an important component of e-governance (Borins, 2002) and had to be addressed by the RSD. At the time, only one brand of dedicated video conferencing units provided the necessary video transmission and "on-board" encryption standards. Communications policies were developed for other collaborative efforts among field offices. At first, coordinating schedules was problematic since field offices could not simply e-mail clients names to each other when coordinating appointments; they would first have to "sanitize" the names in order to meet Health Insurance Portability and Accountability Act (HIPAA) privacy requirements.

\section{Finding Five: Organizational And Communications Challenges}

Once the VCT infrastructure was in place, the RSD also had to work to overcome various organizational and communication barriers. Successful program implementation within organizational settings often requires "buy in," and a shift in organizational norms and culture. Initially, the RSD helped formulate a "business plan" to guide the implementation process and to identify and address issues in the early stages of deployment, including internal coordination and scheduling of VCT appointments, the redistribution of FTEs, and communications policies.

Initially, the RSD and agency stakeholders observed occasional resistance among agency employees when implementing this technology into existing bureaucratic structures with pre-existing procedures (and that do not always work in concert with one another). One agency supervisor (personal interview) described the initial VCT deployment process in the SSA and IHS field offices akin to "teaching elephants how to dance," noting that SSA and IHS were old agencies with very established cultures. He also added that facilitating agency-wide "buy-in" for the VCT program during its preliminary stages was like "threading a needle" because bureaucracies are "built to say no" to change. Additionally, one of the VCT project managers overseeing the implementation recounted that the bureaucratic or "organizational timeline" for VCT deployment did always not match the RSD "project timeline," which resulted in a number of implementation delays (personal interview).

Other organizational challenges centered on finances, including discussions over third party reimbursements, staffing and the shifting and coordination of organizational resources to staff the VCT terminals. To this end, one participant credited a few dedicated "entrepreneurs" within the ranks of the SSA and HIS for their efforts in promoting the program, "smoothing over" some of the initial technological "hiccups," and helping to integrate the VCT technology and its use into the organizations' cultures. Strong programmatic support from the regional offices was also instrumental in getting the different agencies and their field offices "talking to each other" (personal interview). Another participant characterized the successful implementation and sustained use of VCT as a result of "passing the right people barrier," noting that the strength of her relationship with the IHS field office was in no small part attributable to the quality and competency of its benefits coordinator (personal interview). However another participant reported that personnel in another smaller field office resisted the VCT implementation from the start and has yet to use the technology to its fullest extent.

In an unanticipated use of the VCT technology, agency personnel and caseworkers were able to utilize the video links to enhance training among agency personnel and healthcare providers through "face-to-face" contact with one another. During the implementation of the VCT technology, another field office saw a significant reduction in its staff due to a number of planned retirements. In an innovative solution, the person who took over the office's VCT operations was able to utilize the VCT technology to accelerate her acclimation to the position by connecting to other VCT sites to receive training from other caseworkers and benefits coordinators. 


\section{CONCLUSIONS}

Scholarly accounts suggest that ultimately "e-government" must provide truly interactive platforms with "public outreach and accountability enhancing features" (West, 2004, p. 17) and horizontal integration with "real one stop shopping for citizens" (Layne, 2001, p. 124). A truly interactive model of e-governance with integrated service provision and "one-stop shopping" will require fundamental changes to bureaucratic organizations (Tat-Kei, 2002), however, the RSD case can serve as a benchmark for future rural health "e-government" initiatives with its interactive platform and horizontal integration of health and social benefits access that crosscut SSA, IHS and the VA, among others.

From a practical perspective, the RSD bridged a critical gap in the delivery of services to those in poor, rural areas and supplemented traditional modes of public service delivery in these Great Plains states. Indeed,

Mail-based services require the applicant to have the understanding, organization, patience and discipline to independently complete a lengthy and complex paper application. For individuals who do not possess these qualities, mail-based services are of limited utility. Telephone-based services require that an applicant have reliable, economical, and extended access to telephone service, in a private and quite location. If the applicant's home does not provide a good setting for telephone-based services, they are not a viable option. Visits to...offices require reliable transportation, money for travel, time for travel, and possibly care for other family members. The neediest citizen often lacks some or all of these resources, thus removing office visits as viable options.... (SSA, 2007 quoted in RSD, 2007, 3).

The foregoing case of the RSDs implementation of VSD in the IHS and SSA contributes to a better understanding of how to leverage an interactive video platform to provide government healthcare reimbursement services to underrepresented groups in rural contexts. This examination can also help inform entrepreneurs in other government agencies who are interested in providing services to their stakeholders through similar delivery models as well as health care providers who are interested in accessing remote services for their clients.

\section{AUTHOR INFORMATION}

Dana Michael Harsell, Ph.D. is an Assistant Professor of Political Science and Public Administration at the University of North Dakota. He teaches courses in American government, public administration and management, and state and local government. Harsell holds a BA in political science and psychology and an MA in political science, both from the University of Montana, and a $\mathrm{PhD}$ in political science from Syracuse University. Dana's research interests include public management, government performance and accountability, public service provision, and teaching methods in political science and public administration. E-mail: dharsell@business.und.edu

Christine C. Harsell, MS, RN, ANP-BC is a Clinical Assistant Professor in the College of Nursing and Co-Director of the Gerontological Nursing Track at the University of North Dakota. She holds a Master's degree from Syracuse University and teaches courses in professional role development, illness management and evidence based practice. Her research interests include primary care of adults, rural health and access to care, nursing policy and education. Harsell also practices as an adult nurse practitioner in a rural community health center in North Dakota. E-mail: christineharsell@mail.und.edu

Robert S. Wood, Ph.D. is an Associate Professor of Political Science and Public Administration at the University of North Dakota, a Senior Research Associate at the Bureau of Governmental Affairs at UND and an Associate Fellow at the Center for American Politics and Public Policy at the University of Washington. His teaching interests include public policy processes, American national politics, environmental and health policy, bureaucracy and public administration. In addition to public policy processes, Professor Wood's research interests include bureaucratic behavior and regulatory policy. E-mail: professorwood@gmail.com 


\section{REFERENCES}

1. Aberbach, J. D., \& Rockman, B. A. (2002). Conducting and coding elite interviews. PS: Political Science and Politics, 35(4), 673-676.

2. Borins S. (2002). On the frontiers of electronic governance: A report on the United States and Canada. International Review of Administrative Science, 68, 199-211.

3. Carr, P., \& Kefalas, M. J. (2009). Hollowing out the middle: The rural brain drain and what it means for America. Boston, MA: Beacon Press.

4. Felix, H., Shepard, J., \& Stewart M. K. (2003). Recruitment of rural health care providers: A regional recruiter strategy. The Journal of Rural Health, 19: 340-346.

5. Gamm, L. D., Hutchison, L. L., Dabney, B. K., \& Dorsey A. M. (2003). Rural healthy people 2010: A companion document to healthy people 2010, vol. 1. College Station, Texas: The Texas A\&M University System Health Science Center, School of Rural Public Health, Southwest Rural Health Research Center.

6. Layne, K., \& Jungwoo, L. (2001). Developing fully functional e-government: A four stage model. Government Information Quarterly, 18, 121-136.

7. Silcock, R. (2001).What is e-government? Parliamentary Affairs, 54, 88-101.

8. Stake, R. E. (1978). The case study method in social inquiry. Educational Researcher, 7(2), 5-8.

9. Stevens, G. D., Seid, M., \& Halfon, N. (2006). Enrolling vulnerable, uninsured but eligible children in public health insurance: Association with health status and primary care access. Pediatric, 117(4), e751e759.

10. Stuber, J., \& Bradley, E. (2005). Barriers to Medicaid enrollment: Who is at risk? American Journal of Public Health, 95, 292-298.

11. Tat-Kei Ho, A. (2002). Reinventing local governments. Public Administration Review, 62(4), 434-444.

12. The University of North Dakota Center for Rural Service Delivery. (2007). Community challenges and opportunities. Grand Forks, ND: The University of North Dakota Center for Rural Service Delivery.

13. US Government Accountability Office. (2006). Telecommunications: broadband deployment is extensive throughout the United States, but it is difficult to assess the extent of deployment gaps in rural areas. Washington, DC: US Government Accountability Office, GAO-06-426.

14. US Government Accountability Office. (2008). Medicare and Medicaid: CMS and state efforts to interact with the Indian Health Service and Indian Tribes. Washington, DC: US Government Accountability Office, GAO-08-724.

15. US Indian Health Service. (2006). Facts on Indian health disparities. Washington, DC: US Indian Health Service. Retrieved from http://info.ihs.gov/Files/DisparitiesFacts-Jan2006.pdf.

16. West, D. M. (2004). E-Government and the transformation of service delivery and citizen attitudes. Public Administration Review, 64(1):15-27.

17. Yin, R. K. (2003a). Case study research: Design and methods (3rd ed.). Thousand Oaks, CA: Sage Publications.

18. Yin, R.K. (2003b). Applications of case study research (2nd ed.). Thousand Oaks, CA: Sage Publications.

19. Yin, R.K. (2009). Case study research: Design and methods (4th ed.). Thousand Oaks, CA: Sage Publications. 
NOTES 